\title{
Constraints on Kinetically Modified Inflation from WMAP5
}

\author{
Larissa Lorent* and Jérôme Martin话 \\ Institut d'Astrophysique de Paris, UMR 7095-CNRS, \\ Université Pierre et Marie Curie, 98bis boulevard Arago, 75014 Paris, France \\ Christophe Ringevat \\ Theoretical and Mathematical Physics Group, Centre for Particle Physics and Phenomenology, \\ Louvain University, 2 Chemin du Cyclotron, 1348 Louvain-la-Neuve, Belgium
}

(Dated: July 15, 2008)

\begin{abstract}
Single field inflationary models with a non-minimal kinetic term (also called k-inflationary models) can be characterised by the so-called sound flow functions, which complete the usual Hubble flow hierarchy. These parameters appear in the primordial power spectra of cosmological perturbations at leading order and, therefore, affect the resulting Cosmic Microwave Background (CMB) anisotropies. Using the fifth year Wilkinson Microwave Anisotropy Probe (WMAP5) data, we derive the marginalised posterior probability distributions for both the sound and Hubble flow parameters. In contrast to the standard situation, these parameters remain separately unbounded, and notably there is no longer any upper limit on $\epsilon_{1}$, the first Hubble flow function. Only special combinations of these parameters, corresponding to the spectral index and tensor-to-scalar ratio observables, are actually constrained by the data. The energy scale of k-inflation is nevertheless limited from above to $H_{\mathrm{inf}} \leq 6 \times 10^{-6} \mathrm{~m}_{\mathrm{Pl}}$ at two-sigma level. Moreover, for the sub-class of Dirac-Born-Infeld models, by considering the non-gaussianity bounds on the sound speed, we find a weak limit $\epsilon_{1}<0.08$ at $95 \%$ confidence level.
\end{abstract}

PACS numbers: $98.80 . \mathrm{Cq}, 98.70 . \mathrm{Vc}$

\section{INTRODUCTION}

Accelerated expansion of the early Universe in scalar field models is usually achieved by domination of the field's potential over its kinetic energy. However, it has been shown that inflation can also be supported through modifications of the kinetic term, these models being known as "k-inflation" scenarios [1]. Assuming the gravity sector to be described by General Relativity [the signature of the metric tensor being $(-,+,+,+)]$, the action describing such a "k-inflaton" $\varphi\left(x^{\mu}\right)$ reads

$$
S=\frac{1}{2 \kappa} \int \mathrm{d}^{4} x \sqrt{-g}[R+2 \kappa P(X, \varphi)],
$$

where $\kappa \equiv 8 \pi / m_{\mathrm{P} 1}^{2}$ and where $X$ is defined by the following expression

$$
X \equiv-\frac{1}{2} g^{\mu \nu} \partial_{\mu} \varphi \partial_{\nu} \varphi
$$

The quantity $P(X, \varphi)$ is any acceptable functional (see below) for the scalar field Lagrangian. For instance, the canonical scalar field model is recovered for $P(X, \varphi)=$ $X-V(\varphi)$, where $V(\varphi)$ is the scalar field potential.

The theory of k-inflation is interesting not only because it enlarges the class of inflationary models that one can consider but also because this is precisely this type of

\footnotetext{
*Electronic address: lorenz@iap.fr

${ }^{\dagger}$ Electronic address: jmartin@iap.fr

‡Electronic address: christophe.ringeval@uclouvain.be
}

scenarios that emerges from string theory. This is the case of the so-called Dirac-Born-Infeld (DBI) scenarios of brane inflation, which have recently received particular attention as effective field theory realizations of inflation within string theory $2,23,4,5,6,6,7,8,9]$. The DBI inflaton typically represents the distance (i.e., an open string mode) between branes in a higher-dimensional bulk, and the Lagrangian functional is given by the determinant of the induced metric along the moving brane,

$$
P^{\mathrm{DBI}}(X, \varphi)=-T(\varphi) \sqrt{1-\frac{2 X}{T(\varphi)}}+T(\varphi)-V(\varphi) .
$$

Here, $T(\varphi)$ is a warping function encoding the bulk geometry, and $V(\varphi)$ is the interaction potential between the branes, assembled from contributions of various origin.

In general, a model of k-inflation is sensible if the function $P(X, \varphi)$ satisfies the two following conditions. Firstly, $P_{X}>0$, coming from the requirement that the Hamiltonian is bounded from below and, secondly, $P_{, X}+2 X P_{, X X}>0$, which is necessary in order for the Cauchy problem to be well-posed, see for instance Ref. [10]. Let us notice that the DBI models satisfy these requirements. Moreover, these two conditions play an important role at the perturbative level. Indeed, all kinetically modified inflationary models share the common feature that the "speed of sound"

$$
c_{\mathrm{s}}^{2} \equiv \frac{P_{, X}}{P_{, X}+2 X P_{, X X}},
$$

is generically different from the speed of light and may be space-time dependent. Clearly, the two above-mentioned 
inequalities guarantee that $c_{\mathrm{s}}^{2}>0$. This is relevant for the treatment of cosmological perturbations. Indeed, for a flat Friedmann-Lemaître-Robertson-Walker (FLRW) universe, it was shown in Ref. [11], that the MukhanovSasaki mode function $\mu_{\mathrm{S}}(k, \eta)=\zeta \sqrt{2 \kappa \epsilon_{1}} / c_{\mathrm{s}}$ ( $\zeta$ being the comoving curvature perturbation) satisfies the modified equation of motion

$$
\mu_{\mathrm{S}}^{\prime \prime}+\left[c_{\mathrm{s}}^{2} k^{2}-\frac{\left(a \sqrt{\epsilon_{1}} / c_{\mathrm{s}}\right)^{\prime \prime}}{a \sqrt{\epsilon_{1}} / c_{\mathrm{s}}}\right] \mu_{\mathrm{s}}=0 .
$$

Derivatives are with respect to conformal time $\eta, a(\eta)$ is the FLRW scale factor and $\epsilon_{1}=-\mathrm{d} \ln H / \mathrm{d} \ln a$ is the first Hubble flow function ( $H$ being the Hubble parameter). In addition to the induced modification of the perturbations' effective potential, $c_{\mathrm{s}}$ also appears as their propagation speed. Let us notice that for DBI inflation, Eqs. (3) and (4) give

$$
c_{\mathrm{s}}=\sqrt{1-\frac{\varphi^{\prime 2}}{a^{2} T(\varphi)}},
$$

and therefore ensure that $c_{\mathrm{s}} \leq 1$. In the following, we will only consider the k-inflation models for which $c_{\mathrm{s}} \leq 1$ (see, however, Refs. 12, 13, 14, 15, 16, 17]).

In this paper, we are interested in constraining the shape of the scalar and tensor primordial power spectra for all the kinetically modified models admitting an observable "slowly-evolving" phase. To this end, we assume the existence of a background solution supporting a long enough inflationary era to solve the FLRW problems.

The fact that $c_{\mathrm{s}}$ is time-dependent and appears in front of the $k^{2}$ term in Eq. (5) has important consequences and brings new complications into the problem. In particular, one can no longer use the standard techniques consisting in solving the mode equation in terms of Bessel functions. In the case of $\mathrm{k}$-inflation, one is therefore forced to rely on a new method of approximation. This is done in Ref. 18] and in Ref. [19], this last article making use of the Wentzel-Kramers-Brillouin (WKB)/uniform approximation [20, 21, 22]. In this manner, one can solve Eq. (5D) perturbatively in terms of a double hierarchy of functions [7, 18, 19, 23, 24] defined by

$$
\begin{array}{ll}
\epsilon_{i+1}=\frac{\mathrm{d} \ln \left|\epsilon_{i}\right|}{\mathrm{d} \ln a}, & \epsilon_{0}=\frac{H_{\mathrm{ini}}}{H}, \\
\delta_{i+1}=\frac{\mathrm{d} \ln \left|\delta_{i}\right|}{\mathrm{d} \ln a}, & \delta_{0}=\frac{c_{\mathrm{sini}}}{c_{\mathrm{s}}} .
\end{array}
$$

The $\epsilon_{i}$ are the usual Hubble flow functions introduced in Refs. [25, 26, 27], while the $\delta_{i}$ are their equivalent based on the sound speed and therefore encode its rate of change. At first order in these parameters, the scalar and tensor primordial power spectra ${ }^{1}$ can be expressed

\footnotetext{
${ }^{1}$ For single field models, $\zeta$ is a conserved quantity on "super-sonic" length scales. As in the standard case [28], this allows us to propagate the primordial spectrum from the end of inflation to the time of decoupling.
}

as 19

$$
\begin{aligned}
\mathcal{P}_{\zeta} & =\frac{H_{\mathrm{inf}}^{2}}{\pi m_{\mathrm{Pl}}^{2} \epsilon_{1} c_{\mathrm{s}}}\left[1-2(D+1) \epsilon_{1}-D \epsilon_{2}+(D+2) \delta_{1}\right. \\
& \left.-\left(2 \epsilon_{1}+\epsilon_{2}-\delta_{1}\right) \ln \frac{k}{k_{*}}\right], \\
\mathcal{P}_{h} & =\frac{16 H_{\mathrm{inf}}^{2}}{\pi m_{\mathrm{P} 1}^{2}}\left[1-2\left(D+1-\ln c_{\mathrm{s}}\right) \epsilon_{1}-2 \epsilon_{1} \ln \frac{k}{k_{*}}\right] .
\end{aligned}
$$

The wavenumber $k_{*}$ is a pivot scale around which these power spectra are expanded, see Ref. [19]. All the functions appearing in this equation have to be evaluated at the conformal time $\eta_{*}$ at which this pivot mode crossed the sound horizon, namely the solution of

$$
k_{*} \eta_{*}=-\frac{1}{c_{\mathrm{s}}\left(\eta_{*}\right)} .
$$

Notice that although the tensor perturbations are not affected by the non-minimal kinetic terms in the scalar sector, $c_{\mathrm{s}}$ nevertheless appears in $\mathcal{P}_{h}$ due to the evaluation of the flow functions at the sound horizon crossing time instead of Hubble crossing. In Eq. (8), $D=1 / 3-\ln 3 \simeq$ -0.76 is a constant coming from the uniform approximation, and an overall constant factor of $18 \exp (-3)$ has been absorbed into $H_{\mathrm{inf}}^{2}$ [22]. From Eq. (8) we can read off the spectral indices to first order,

$$
n_{\mathrm{S}}-1=-2 \epsilon_{1}-\epsilon_{2}+\delta_{1}, \quad n_{\mathrm{T}}=-2 \epsilon_{1},
$$

and the k-inflation tensor to scalar ratio $r=16 \epsilon_{1} c_{\mathrm{s}}$.

As expected, the power spectra in Eq. (8) are not the same than those obtained for a canonically normalised scalar field due to the presence of the two sound flow parameters $c_{\mathrm{s}}$ and $\delta_{1}$. Notice that in the limit $c_{\mathrm{s}}=1-$ $\mathcal{O}\left(\epsilon_{i}\right)$, one has $\delta_{1}=\mathcal{O}\left(\epsilon_{i}^{2}\right)$ and the above spectra only differ from their usual analogue at second order in the Hubble flow functions 29].

In the following, we compare the induced Cosmic Microwave Background (CMB) anisotropies seeded by these power spectra to the WMAP5 data [30, 31, 32, 33, 34, 35]. Using Markov-Chains-Monte-Carlo (MCMC) methods, we extract the posterior marginalised probability distributions on the sound and Hubble flow parameters and discuss the physical consequences. Moreover, as shown in Refs. [24, 36, 37, 38], kinetically modified inflation models may induce a large amount of nongaussianities in the statistics of the CMB anisotropies, usually measured in terms of the $f_{\mathrm{NL}}$ parameter [39]. The expected value for $f_{\mathrm{NL}}$ strongly depends on the model at hand: as shown in Ref. 24], $f_{\mathrm{NL}}$ for equilateral configurations demands the knowledge of the third $X$-derivative of $P(X, \varphi)$ in addition to the sound and Hubble flow functions. For these reasons, we have chosen to split our analysis into two parts. In the first section, we do not consider the non-gaussianity constraints, and our results are only based on the observed two-point functions of the $\mathrm{CMB}$ temperature and polarisation anisotropies. In the 
second part, we implement the WMAP5 bounds on $f_{\mathrm{NL}}$ as prior knowledge on the sound speed values in the restricted class of DBI models: for those, we indeed have the universal expression [24, 40, 41]

$$
f_{\mathrm{NL}}=\frac{35}{108}\left(1-\frac{1}{c_{\mathrm{s}}^{2}}\right)+\mathcal{O}\left(\epsilon_{i}, \delta_{i}\right)
$$

From now on, $f_{\mathrm{NL}}$ is implicitly assumed to refer to equilateral configurations, the "local" estimator being $f_{\mathrm{NL}}^{\text {local }} \sim \mathcal{O}\left(\epsilon_{i}, \delta_{i}\right)$ and well below the current detection thresholds, see Ref. [24, 35, 37].

\section{CMB POWER SPECTRA}

We have used a modified version of the CAMB code [42] to compute the $\mathrm{CMB}$ temperature and polarisation anisotropies seeded by the scalar and tensor primordial power spectra of Eqs. (8). Comparison with the WMAP data is then performed by exploring the full parameter space, including the standard cosmological parameters, through MCMC Bayesian methods implemented in the COSMOMC package [43, 44]. The likelihood estimator is provided by the WMAP team [34].

\section{A. Cosmological model}

The assumed cosmological model is a flat $\Lambda$ CDM universe involving four cosmological base parameters (with priors chosen from the posteriors of the previous CMB experiments): the number density of baryons $\Omega_{\mathrm{b}}$, of cold dark matter $\Omega_{\mathrm{dm}}$, the reionization optical depth $\tau$, and $\theta$ which measures the ratio of the acoustic horizon to the angular diameter distance (see Ref. [43]). As performed by the WMAP team, we have now included the lensing of the CMB power spectra and performed a marginalisation over the Sunyaev-Zel'dovich amplitude $A_{\mathrm{sz}}$, in unit of the analytical model of Ref. [45] and with a flat prior in [0,2]. For the sake of clarity, and to allow comparison with previous works [46], we have, at this stage, not considered more data sets than the WMAP 5 data, the Hubble Space Telescope (HST) constraint $\left(H_{0}=72 \pm 8 \mathrm{~km} / \mathrm{s} / \mathrm{Mpc}\right.$ [4] $)$ and a top hat prior on the age of the universe between 10 Gyrs and 20 Gyrs. Notice that we have chosen the pivot scale to be in the middle of the observable range, namely $k_{*}=0.05 \mathrm{Mpc}^{-1}[48]$.

\section{B. Observable primordial parameters and priors}

It is important to notice that the new parameters appearing in the primordial power spectra of Eqs. (8) induce new degeneracies. In particular, and contrary to the usual case, $\epsilon_{1}$ is no longer uniquely linked to the tensor to scalar ratio due to the presence of $c_{\mathrm{s}}$. Therefore, the product $\epsilon_{1} c_{\mathrm{S}}$ is a better motivated observable parameter in k-inflation. Its order of magnitude being a priori
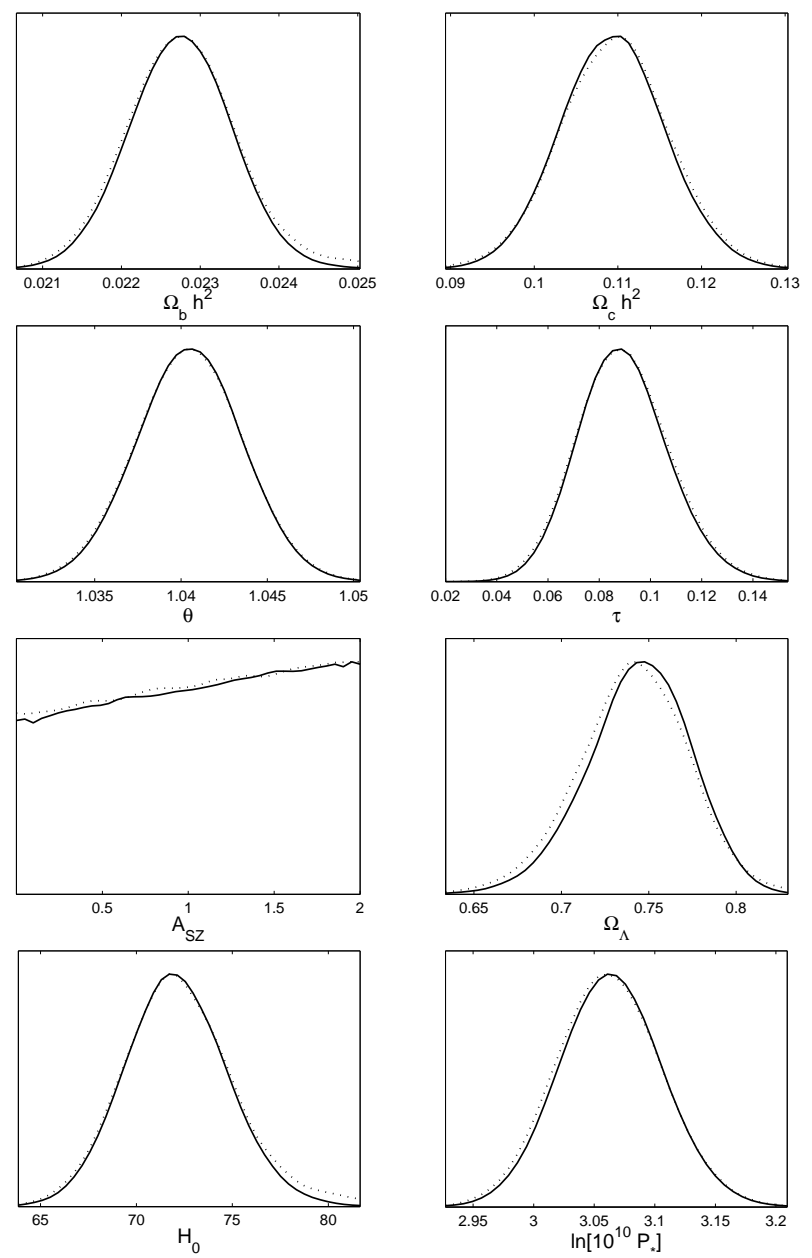

FIG. 1: One dimensional marginalised posterior probability distribution (solid) and mean likelihoods (dotted) for the base and derived cosmological parameters, given the WMAP5 data and the k-inflation power spectra of Eq. (8).

unknown, we have chosen a uniform logarithmic prior ${ }^{2}$ : $-10<\log \left(\epsilon_{1} c_{\mathrm{s}}\right)<-0.5$ (Jeffreys' prior).

Apart from the overall amplitude, $\mathcal{P}_{\zeta}(k)$ in Eqs. (8) has exactly the same functional form than the canonical scalar slow-roll power spectrum at first order upon the replacement $\epsilon_{1} \rightarrow \epsilon_{1}-\delta_{1}$, and $\epsilon_{2} \rightarrow \epsilon_{2}+\delta_{1}$. In particular, this is the case for the spectral index $n_{\mathrm{S}}$ in Eq. (10). As a result, $\epsilon_{1}, \epsilon_{2}$ and $\delta_{1}$ are fully degenerated and there are only two observable parameters, namely $\left(\epsilon_{1}-\delta_{1}\right)$ and $\left(\epsilon_{2}+\delta_{1}\right)$, for which we have chosen a uniform prior in $[-0.3,0.3]$. This important point already shows that, at the scalar perturbation level, there is no chance of constraining $\delta_{1}$ separately, neither $\epsilon_{1}$ nor $\epsilon_{2}$; this is significantly different from the usual slow-roll constraints [46, 49, 50, 51]. However, considering the pri-

\footnotetext{
${ }^{2}$ Recall that we are considering the case $c_{\mathrm{S}}^{2} \leq 1$.
} 

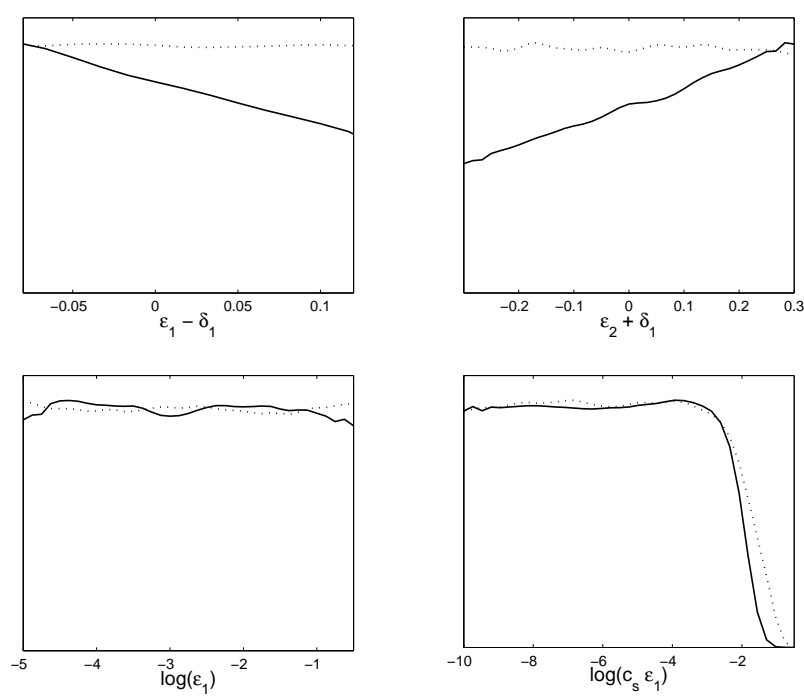

FIG. 2: One dimensional marginalised posterior probability distribution (solid) and mean likelihood (dotted) for the sampled sound and Hubble flow parameters given the WMAP5 data. Only $\log \left(\epsilon_{1} c_{\mathrm{S}}\right)$ is upper limited, this parameter fixing the tensor-to-scalar ratio. Notice in particular that $\epsilon_{1}$ is not constrained. The parameters $\left(\epsilon_{1}-\delta_{1}\right)$ and $\left(\epsilon_{2}+\delta_{1}\right)$ are, however, correlated (see Fig. 3).

mordial tensor power spectrum $\mathcal{P}_{h}(k)$, one sees that $\epsilon_{1}$ is still uniquely linked with the tensor spectral index $n_{\mathrm{T}}$ [see Eq. (10)]. As one may expect, it is unconstrained by the data at present, and so should be $\epsilon_{1}$. We have however included it for completeness as the third primordial parameter in the MCMC exploration with a logarithmic prior in $-5 \leq \log \epsilon_{1} \leq-0.5$. Finally, the overall amplitude of the CMB anisotropies is fixed by $P_{*}=\mathcal{P}_{\zeta}\left(k_{*}\right)$ for which we have chosen the usual uniform prior distribution on $\ln \left(10^{10} P_{*}\right)$ in $[2.7,4]$.

\section{Results}

The MCMC exploration has been stopped according to the $\mathrm{R}$-statistics implemented in COSMOMC [43, 52] as soon as the variances between the different chains agree at a few percent. The chains contain 800000 samples and lead to the marginalised posterior probability distributions represented in Fig. 11 to Fig. 3. The base cosmological parameters associated with the $\Lambda$ CDM universe are compatible with the recent results [35], although our primordial power spectra are different.

Fig. 2 shows the posterior probability distributions (solid curves) for each of the primordial parameters we are interested in. As we expected, there is no bound on $\log \epsilon_{1}$ and the posterior is just given by the prior. On the other hand, the maximum amount of tensor modes
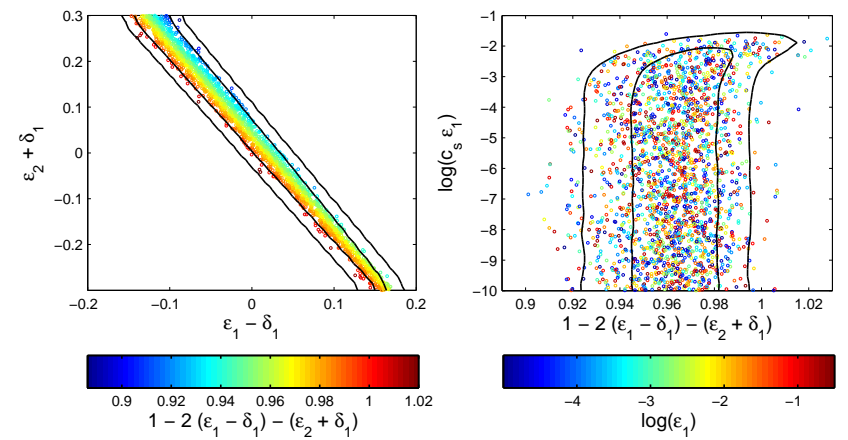

FIG. 3: Two dimensional marginalised posteriors (point density) together with the one and two-sigma contours. Although $\left(\epsilon_{1}-\delta_{1}\right)$ and $\left(\epsilon_{2}+\delta_{1}\right)$ are not bounded, they are strongly correlated to give acceptable values of the spectral index (left panel). The right panel exhibits the correlations between $\log \left(\epsilon_{1} c_{\mathrm{S}}\right)$ and $n_{\mathrm{S}}=1-2\left(\epsilon_{1}-\delta_{1}\right)-\left(\epsilon_{2}+\delta_{1}\right)$.

in the CMB power spectra gives an upper limit on the parameter $\log \left(\epsilon_{1} c_{\mathrm{s}}\right)$. Its posterior is indeed upper limited and at $95 \%$ of confidence, one finds

$$
\log \left(\epsilon_{1} c_{\mathrm{s}}\right) \leq-2.3
$$

Notice that the above upper limit is stronger than the ones reported in the recent literature [35, 53]. This effect comes from our Jeffreys' prior choice on $\epsilon_{1} c_{\mathrm{s}}$, which favours low values of the parameter [44], and possibly from the new natural choice of the sampling parameters $\left(\epsilon_{1}-\delta_{1}\right)$ and $\left(\epsilon_{2}+\delta_{1}\right)$. Let us mention that this prior choice is motivated by our ignorance on the scale at which tensor perturbations contribute to the CMB, a priori ${ }^{3}$. The slight discrepancies appearing in between the posterior and the mean likelihood for this parameter signal some correlations with other parameters [43, 54]. Also, both $\left(\epsilon_{1}-\delta_{1}\right)$ and $\left(\epsilon_{2}+\delta_{1}\right)$ exhibit unbounded distributions, and their mean likelihood (dotted curves) differs from the marginalised posterior. The two-dimensional marginalised probability distribution in the plane $\left(\epsilon_{1}-\delta_{1}, \epsilon_{2}+\delta_{1}\right)$ is plotted in Fig. 3 and clearly exhibits the above-mentioned correlations. The physical interpretation is straightforward: the "correlation strip" is given by the allowed values of the scalar spectral index $n_{\mathrm{S}}$ [see Eq. (10)]. The barely visible thickening of this strip comes from some slight correlations with the parameter $P_{*}$, and then $A_{\mathrm{sz}}$, due to the functional form of the power spectra. Finally, as can be seen in Fig. 3, large values of $\log \left(\epsilon_{1} c_{\mathrm{S}}\right)$ are also degenerated with the spectral index $n_{\mathrm{S}}$ in a way reminiscent of the corresponding correlation between tensor-to-scalar ratio and spectral index in standard inflation.

\footnotetext{
${ }^{3}$ From a flat prior on $\epsilon_{1} c_{\mathrm{S}}$, we obtain $\epsilon_{1} c_{\mathrm{S}}<0.023$ at two-sigma level (by importance sampling).
} 

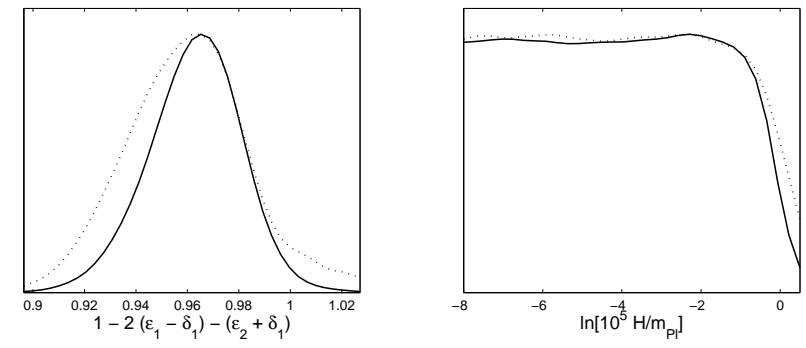

FIG. 4: Marginalised posterior probability distribution for the derived primordial parameters $n_{\mathrm{S}}$ and $H_{\text {inf }}$ obtained by importance sampling.

In Fig. 4, we have used importance sampling to reconstruct the probability distribution of $n_{\mathrm{S}}$ and $\ln \left(10^{5} H_{\text {inf }} / m_{\mathrm{Pl}}\right)$. The probability distribution for $n_{\mathrm{S}}$ ends up being compatible with the one found by assuming a power law primordial spectrum [35]. At $95 \%$ of confidence, one gets

$$
0.003 \leq 2\left(\epsilon_{1}-\delta_{1}\right)+\left(\epsilon_{2}+\delta_{1}\right) \leq 0.075 .
$$

This limit favours a "red tilted" scalar power spectrum, at two-sigma level, in presence of tensor modes and varying sound speed ${ }^{4}$. The probability distribution associated with $H_{\text {inf }}$ is only upper limited since this parameter is fully degenerated with $\epsilon_{1} c_{\mathrm{s}}$ in fixing the amplitude of the primordial scalar power spectrum [see Eq. [8] ]. The prior distribution on $H_{\text {inf }}$ is logarithmically uniform and induced by our Jeffreys' prior on $\epsilon_{1} c_{\mathrm{s}}$ and $P_{*}$. This is convenient since the energy scale of inflation is also unknown a priori. We find

$$
\ln \left(10^{5} \frac{H_{\mathrm{inf}}}{m_{\mathrm{Pl}}}\right) \leq-0.59
$$

at $95 \%$ of confidence.

\section{INCLUDING NON-GAUSSIANITY}

In this section, we use the two-sigma WMAP5 bound on the $f_{\mathrm{NL}}$ parameter associated with "equilateral configurations" in Fourier space [35],

$$
-151 \leq f_{\mathrm{NL}} \leq 253
$$

As already mentioned, the predicted value of $f_{\mathrm{NL}}$ for $\mathrm{k}$ inflation models is not universal and depends on the explicit form of the third derivative of $P(X, \varphi)$ with respect to $X$. As a result, we have chosen to limit our analysis to the sub-class of DBI models: for those, Eq. (11) can be used.

\footnotetext{
4 This is, however, prior dependent; from a flat prior on $\epsilon_{1} c_{\mathrm{S}}$, the bounds become $[-0.024,0.063]$.
}
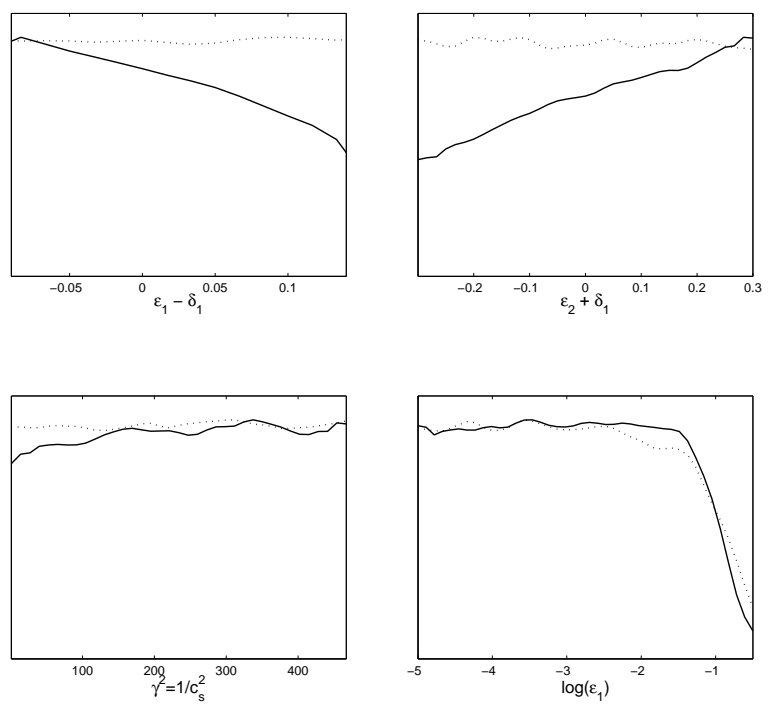

FIG. 5: Marginalised posterior probability distribution for the DBI primordial parameters by including the non-gaussianity bounds on $f_{\mathrm{NL}}$. The first Hubble flow parameter $\epsilon_{1}$ is now upper limited.

\section{A. New priors}

A straightforward implementation of the nongaussianity bound is to include it as prior knowledge on the acceptable $c_{\mathrm{s}}$ values. In fact, this occurs via the socalled "Lorentz factor" $\gamma^{2} \equiv 1 / c_{\mathrm{s}}^{2}$ which enters into the expression (11). Since its order of magnitude is now fixed, we have chosen to sample the parameter space over $\gamma^{2}$, instead of $\log \left(\epsilon_{1} c_{\mathrm{s}}\right)$, with an uniform prior over [1,467]. All the other sampling parameters and priors remain the same as in Sect. II] as well as the cosmological model.

\section{B. Results}

The marginalised posterior probability distributions associated with the base and derived cosmological parameters are essentially the same as in the previous section and we do not discuss them any further (see Fig. 11). The posteriors of the parameters appearing in the primordial power spectra are plotted in Fig. 5 .

In fact, the non-gaussianity prior imposed on $\gamma^{2}$ breaks the degeneracies we encountered previously between $\epsilon_{1}$ and $c_{\mathrm{s}}$, and which motivated the use of $\log \left(\epsilon_{1} c_{\mathrm{s}}\right)$ as a sampling parameter. The sound speed being now limited from below, the tensor-to-scalar ratio becomes sensitive to the first Hubble flow parameter and we find a "weak" upper bound on $\log \epsilon_{1}$. At $95 \%$ of confidence,

$$
\log \epsilon_{1} \leq-1.1
$$



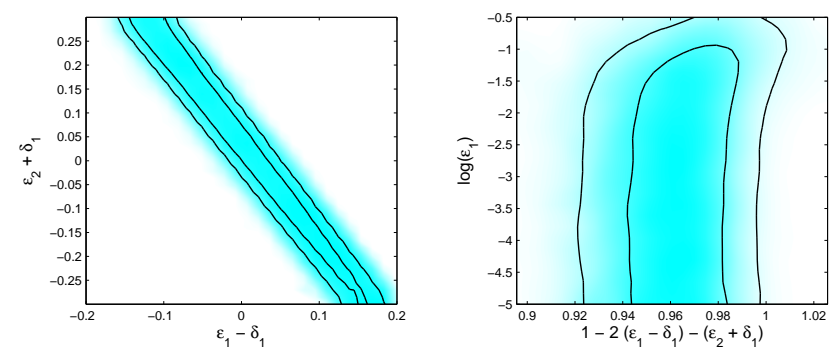

FIG. 6: One and two-sigma contours of the two dimensional marginalised posteriors for correlated pairs of primordial parameters. The shading traces the two-dimensional mean likelihood. The right panel shows that the non-gaussianity bounds on $f_{\mathrm{NL}}$ induces an upper bound on the first Hubble flow parameter $\epsilon_{1}$.

On the other hand, $\left(\epsilon_{1}-\delta_{1}\right)$ and $\left(\epsilon_{2}+\delta_{1}\right)$ are still unbounded but correlated along the acceptable values of the spectral index (see Fig. 6). As a result, $\delta_{1}$ remains unconstrained, and so does $\epsilon_{2}$. The posteriors for the spectral index and the energy scale of inflation $H_{\text {inf }}$ appear to be relatively insensitive to the inclusion of the non-gaussianity bound. Their respective two-sigma limits are still given by Eq. (13) and Eq. (14).

In addition to imposing strong bounds on $c_{\mathrm{s}}$ for DBI models, the non-gaussianity limits of Eq. (15) allow disambiguation of the first Hubble flow parameter, and thus, some inference on the evolution of the Hubble parameter during inflation. It is clear that the limit of Eq. (16) is not very tight, and quite close to the values acceptable for the "slowly-evolving" limit. However, improving the bounds on $f_{\mathrm{NL}}$ will directly improve the determination of the acceptable $\log \left(\epsilon_{1}\right)$ values: one may therefore expect tighter constraints from the next generation of $\mathrm{CMB}$ experiments.

\section{CONCLUSION}

We have used the WMAP fifth year data to constrain all single field k-inflationary models admitting a phase of "slow-rolling" evolution during the generation of the cosmological perturbations.

The probability distributions of the Hubble and sound flow parameters, $\epsilon_{i}$ and $\delta_{i}$, evaluated when the scalar perturbations crossed the sound horizon, are significantly different than the ones associated with canonically normalised single field models. The presence of two additional parameters in the primordial power spectra, $c_{\mathrm{s}}$ and $\delta_{1}$, leads to new degeneracies in the parameter space: there is no longer any constraint on $\epsilon_{1}$ and $\epsilon_{2}$ separately, neither on $c_{\mathrm{s}}$ or $\delta_{1}$ alone. The energy scale of inflation is nevertheless upper limited: $H_{\mathrm{inf}} / m_{\mathrm{P} 1} \leq 6 \times 10^{-6}$ at $95 \%$ of confidence. In fact, only two particular combinations of the primordial parameters end up being constrained by the CMB power spectra: the quantity $\epsilon_{1} c_{\mathrm{S}}$ in Eq. (12), which encodes the tensor-to-scalar ratio in the CMB spectra, and $2\left(\epsilon_{1}-\delta_{1}\right)+\left(\epsilon_{2}+\delta_{1}\right)$, which fixes the scalar spectral index in Eq. (13). Under our prior assumptions, Eq. (13) shows that the data favour a "redtilted" scalar power spectrum, at two-sigma level, with tensor modes and varying sound speed.

Implementing the current WMAP5 bound on nongaussianity through the $f_{\mathrm{NL}}$ parameter (for equilateral configurations) breaks some of the above-mentioned degeneracies. For the DBI inflationary models, more than restricting the acceptable values of the sound speed $c_{\mathrm{s}}$, we find that the non-gaussianity bound induces an upper limit on the first Hubble flow parameter: $\log \left(\epsilon_{1}\right)<-1.1$ at two-sigma confidence level.

The present results could be improved by various means. A first step may be to add more data sets, but according to the previous discussion, this will certainly improve only the limits on the spectral index and tensorto-scalar ratio combinations without breaking the degeneracies between the $\epsilon_{i}$ and $\delta_{i}$. Therefore, it is clearly more promising to improve the tests that bring new information on non-gaussianities. We have implemented them for DBI models only through a prior knowledge over $c_{\mathrm{s}}$. Clearly, our approach could be improved by directly evaluating the three-point functions and comparing it to the observed one, provided one can define an tractable likelihood for MCMC exploration. Such an approach is currently technically difficult. Evaluating the three-point functions from CMB data is not trivial (see Appendix A in Ref. 35]); and, from a theoretical point of view, new parameters have to be introduced to quantify the non-gaussian signals in a generic way for k-inflation. Therefore, one faces the risk to add even more degeneracies. On the other hand, with the incoming flow of data on cosmological observables, this represents an interesting challenge for the forthcoming years.

\section{Acknowledgments}

We would like to thank G. Geshnizjani for useful discussions. The computations have been performed on the PLANCK-HFI French Processing Center hosted at the Institut d'Astrophysique de Paris. This work is partially supported by the Belgian Federal Office for Science, Technical and Cultural Affairs, under the Inter-university Attraction Pole grant P6/11. LL acknowledges support through a DAAD PhD scholarship.
[1] C. Armendariz-Picon, T. Damour, and V. F. Mukhanov, Phys. Lett. B458, 209 (1999), hep-th/9904075.
[2] G. R. Dvali and S. H. H. Tye, Phys. Lett. B450, 72 
(1999), hep-ph/9812483.

[3] S. Kachru et al., JCAP 0310, 013 (2003), hepth/0308055.

[4] S. H. Henry Tye, Lect. Notes Phys. 737, 949 (2008), hepth/0610221.

[5] M. P. Hertzberg, M. Tegmark, S. Kachru, J. Shelton, and O. Ozcan, Phys. Rev. D76, 103521 (2007), 0709.0002.

[6] R. Bean, X. Chen, H. V. Peiris, and J. Xu, Phys. Rev. D77, 023527 (2008), 0710.1812.

[7] H. V. Peiris, D. Baumann, B. Friedman, and A. Cooray, Phys. Rev. D76, 103517 (2007), 0706.1240.

[8] R. Bean, S. E. Shandera, S. H. Henry Tye, and J. Xu, JCAP 0705, 004 (2007), hep-th/0702107.

[9] L. Lorenz, J. Martin, and C. Ringeval, JCAP 0804, 001 (2008), 0709.3758.

[10] J.-P. Bruneton and G. Esposito-Farese, Phys. Rev. D76, 124012 (2007), 0705.4043.

[11] J. Garriga and V. F. Mukhanov, Phys. Lett. B458, 219 (1999), hep-th/9904176.

[12] J.-P. Bruneton, Phys. Rev. D75, 085013 (2007), grqc/0607055.

[13] C. Bonvin, C. Caprini, and R. Durrer, Phys. Rev. Lett. 97, 081303 (2006), astro-ph/0606584.

[14] G. Ellis, R. Maartens, and M. A. H. MacCallum, Gen. Rel. Grav. 39, 1651 (2007), gr-qc/0703121.

[15] E. Babichev, V. Mukhanov, and A. Vikman, JHEP 02, 101 (2008), 0708.0561.

[16] C. Bonvin, C. Caprini, and R. Durrer (2007), 0706.1538.

[17] J. U. Kang, V. Vanchurin, and S. Winitzki, Phys. Rev. D76, 083511 (2007), 0706.3994.

[18] W. H. Kinney and K. Tzirakis, Phys. Rev. D77, 103517 (2008), 0712.2043

[19] L. Lorenz, J. Martin, and C. Ringeval (2008).

[20] S. Habib, K. Heitmann, G. Jungman, and C. MolinaParis, Phys. Rev. Lett. 89, 281301 (2002), astro$\mathrm{ph} / 0208443$.

[21] S. Habib, A. Heinen, K. Heitmann, G. Jungman, and C. Molina-Paris, Phys. Rev. D70, 083507 (2004), astro$\mathrm{ph} / 0406134$.

[22] J. Martin and D. J. Schwarz, Phys. Rev. D67, 083512 (2003), astro-ph/0210090.

[23] M. Alishahiha, E. Silverstein, and D. Tong, Phys. Rev. D70, 123505 (2004), hep-th/0404084.

[24] X. Chen, M.-x. Huang, S. Kachru, and G. Shiu, JCAP 0701, 002 (2007), hep-th/0605045.

[25] D. J. Schwarz, C. A. Terrero-Escalante, and A. A. Garcia, Phys. Lett. B517, 243 (2001), astro-ph/0106020.

[26] S. M. Leach, A. R. Liddle, J. Martin, and D. J. Schwarz, Phys. Rev. D66, 023515 (2002), astro-ph/0202094.

[27] D. J. Schwarz and C. A. Terrero-Escalante, JCAP 0408,
003 (2004), hep-ph/0403129.

[28] J. Martin and D. J. Schwarz, Phys. Rev. D57, 3302 (1998), gr-qc/9704049.

[29] D. A. Steer and F. Vernizzi, Phys. Rev. D70, 043527 (2004), hep-th/0310139.

[30] B. Gold et al. (WMAP) (2008), 0803.0715.

[31] R. S. Hill et al. (WMAP) (2008), 0803.0570.

[32] G. Hinshaw et al. (WMAP) (2008), 0803.0732.

[33] M. R. Nolta et al. (WMAP) (2008), 0803.0593.

[34] J. Dunkley et al. (WMAP) (2008), 0803.0586.

[35] E. Komatsu et al. (WMAP) (2008), 0803.0547.

[36] D. Seery and J. E. Lidsey, JCAP 0506, 003 (2005), astro$\mathrm{ph} / 0503692$.

[37] R. Bean, D. J. H. Chung, and G. Geshnizjani (2008), 0801.0742 .

[38] C. S. Gauthier and R. Akhoury (2008), 0804.0420.

[39] E. Komatsu and D. N. Spergel, Phys. Rev. D63, 063002 (2001), astro-ph/0005036.

[40] X. Gao, JCAP 0806, 029 (2008), 0804.1055.

[41] D. Langlois, S. Renaux-Petel, D. A. Steer, and T. Tanaka (2008), 0806.0336.

[42] A. Lewis, A. Challinor, and A. Lasenby, Astrophys. J. 538, 473 (2000), astro-ph/9911177, URL http://camb.info

[43] A. Lewis and S. Bridle, Phys. Rev. D66, 103511 (2002), astro-ph/0205436, URL http://cosmologist.info/cosmomc

[44] R. Trotta (2008), 0803.4089.

[45] E. Komatsu and U. Seljak, Mon. Not. Roy. Astron. Soc. 336, 1256 (2002), astro-ph/0205468.

[46] J. Martin and C. Ringeval, JCAP 0608, 009 (2006), astro-ph/0605367.

[47] W. L. Freedman et al., Astrophys. J. 553, 47 (2001), astro-ph/0012376.

[48] A. R. Liddle, D. Parkinson, S. M. Leach, and P. Mukherjee, Phys. Rev. D74, 083512 (2006), astro-ph/0607275.

[49] V. Barger, H.-S. Lee, and D. Marfatia, Phys. Lett. B565, 33 (2003), hep-ph/0302150.

[50] S. M. Leach and A. R. Liddle, Phys. Rev. D68, 123508 (2003), astro-ph/0306305.

[51] F. Finelli, M. Rianna, and N. Mandolesi, JCAP 0612, 006 (2006), astro-ph/0608277.

[52] A. Gelman and D. Rubin, Statistical Science 7, 457 (1992).

[53] W. H. Kinney, E. W. Kolb, A. Melchiorri, and A. Riotto (2008), 0805.2966.

[54] J. Martin and C. Ringeval, JCAP 0501, 007 (2005), hepph/0405249. 